\title{
The HOSPITAL Score Predicts Potentially Preventable 30-Day Readmissions in Conditions Targeted by the Hospital Readmissions Reduction Program
}

\author{
Robert E. Burke, MD, MS, + Jeffrey L. Schnipper, MD, MPH, $\$$ S Mark V. Williams, MD, /I \\ Edmondo J. Robinson, MD, MBA, MSHP, 9 Eduard E. Vasilevskis, MD, MPH,\#** \\ Sunil Kripalani, MD, MSc,\#†† Joshua P. Metlay, MD, PhD, $+\ddagger$ Grant S. Fletcher, MD, MPH, $\S$ \\ Andrew D. Auerbach, MD, MPH, || || and Jacques D. Donzé, MD, MScł\$ $\$$
}

\begin{abstract}
Background/Objectives: New tools to accurately identify potentially preventable 30 -day readmissions are needed. The HOSPITAL score has been internationally validated for medical inpatients, but its performance in select conditions targeted by the Hospital Readmission Reduction Program (HRRP) is unknown.
\end{abstract}

Design: Retrospective cohort study.

Setting: Six geographically diverse medical centers.

From the *Hospital Medicine and Research Sections, Eastern Colorado Health Care System, DE; $†$ Department of Medicine, Division of General Internal Medicine, University of Colorado School of Medicine, Aurora, $\mathrm{CO} ; \mathrm{BWH}$ Hospitalist Service, Division of General Medicine, Brigham and Women's Hospital; §Harvard Medical School, Boston, MA; $\|$ Center for Health Services Research, University of Kentucky College of Medicine, Lexington, KY; $\uparrow$ Value Institute, Christiana Care Health System, Wilmington, DE; \#Section of Hospital Medicine, Division of General Internal Medicine and Public Health, Vanderbilt University; **VA Tennessee Valley, Geriatric Research, Education and Clinical Center (GRECC); $\dagger \dagger$ Center for Clinical Quality and Implementation Research, Vanderbilt University, Nashville, TN; \$tDivision of General Internal Medicine, Massachusetts General Hospital, Boston, MA; \$§Department of Medicine, Harborview Medical Center, University of Washington, Seattle, WA; || ||Division of Hospital Medicine, University of California, San Francisco, CA; and $\uparrow$ Division of General Internal Medicine, Bern University Hospital, Bern, Switzerland.

R.E.B.is supported by the VA (Career Development Award HX-1796) and NIH/NIA (R03AG050885). E.E.V. is supported by the National Institutes of Health (K23AG040157) and the Geriatric Research, Education and Clinical Center (GRECC). J.D.D. was supported by the Swiss National Science Foundation and the Swiss Foundation for MedicalBiological Scholarships. The content of this article is that of the authors and does not necessarily reflect the views of the Department of Veterans Affairs or National Institutes of Health.

Presented at the 2016 Society of Hospital Medicine National Meeting.

J.L.S. has received grant funding from Sanofi Aventis for an investigatorinitiated study to design and evaluate an intensive discharge and followup intervention in patients with diabetes. The remaining authors declare no conflict of interest.

Reprints: Robert E. Burke, MD, MS, Denver VA Medical Center, 1055 Clermont Street, Denver, CO 80220. E-mail: robert.burke5@va.gov.

Supplemental Digital Content is available for this article. Direct URL citations appear in the printed text and are provided in the HTML and PDF versions of this article on the journal's Website, www.lww-medical care.com.

Copyright (C) 2016 Wolters Kluwer Health, Inc. All rights reserved. ISSN: 0025-7079/17/5503-0285
Participants/Exposures: All consecutive adult medical patients discharged alive in 2011 with 1 of the 4 medical conditions targeted by the HRRP (acute myocardial infarction, chronic obstructive pulmonary disease, pneumonia, and heart failure) were included. Potentially preventable 30-day readmissions were identified using the SQLape algorithm. The HOSPITAL score was calculated for all patients.

Measurements: A multivariable logistic regression model accounting for hospital effects was used to evaluate the accuracy (Brier score), discrimination (c-statistic), and calibration (Pearson goodness-of-fit) of the HOSPITAL score for each 4 medical conditions.

Results: Among the 9181 patients included, the overall 30-day potentially preventable readmission rate was $13.6 \%$. Across all 4 diagnoses, the HOSPITAL score had very good accuracy (Brier score of 0.11 ), good discrimination ( $c$-statistic of 0.68 ), and excellent calibration (Hosmer-Lemeshow goodness-of-fit test, $P=0.77$ ). Within each diagnosis, performance was similar. In sensitivity analyses, performance was similar for all readmissions (not just potentially preventable) and when restricted to patients age 65 and above.

Conclusions: The HOSPITAL score identifies a high-risk cohort for potentially preventable readmissions in a variety of practice settings, including conditions targeted by the HRRP. It may be a valuable tool when included in interventions to reduce readmissions within or across these conditions.

Key Words: patient readmission, score, risk factors, transition of care

(Med Care 2017;55: 285-290)

U nder the Affordable Care Act, the Center of Medicare and Medicaid Services (CMS) calculates average riskadjusted 30-day hospital readmission rates for selected conditions and penalizes hospitals with above-average rates. ${ }^{1,2}$ The amount of the penalty and the number of conditions targeted has expanded as the introduction of the Hospital Readmissions Reduction Program (HRRP) in 2012, affecting $78 \%$ of hospitals nationally in 2015 with projected total penalties of $\$ 428$ million. $^{3}$ 
Given this expansion, hospitals are seeking to reduce readmissions for penalized conditions. Best practices for doing so include identifying a high-risk cohort, comprehensively assessing potentially modifiable readmission risks, and targeting these risks. ${ }^{4-7}$ Progress in this regard has been hindered by lack of a validated tool that could identify potentially preventable readmissions, particularly for conditions targeted by the HRRP. The HOSPITAL score was recently shown to identify patients at risk for potentially preventable readmissions in a cohort of discharged patients across 9 hospitals in 4 different countries. ${ }^{8,9}$ However, its performance specifically on selected conditions targeted by the HRRP is unknown. Establishing its performance for each of these conditions is important as hospitals may not face penalties for each condition, and often wish to institute disease-specific interventions to reduce readmissions. ${ }^{10}$ If the HOSPITAL score has reasonable performance for these conditions, its international validation and ability to exclude nonpreventable readmissions may hold promise for hospitals and clinicians intervening to reduce readmissions. We sought to validate the HOSPITAL score for predicting potentially preventable readmissions within each of the medical conditions targeted by the HRRP, in a multisite sample of hospital discharges.

\section{METHODS}

\section{Study Design and Participants}

This was a retrospective cohort study of medical inpatients discharged alive between January 1, 2011 and December 31, 2011 from 6 hospitals in the United States: San Francisco General Hospital, University of California, San Francisco, CA; Hospital of the University of Pennsylvania, Philadelphia, PA; Harborview Medical Center at the University of Washington, Seattle, WA; Vanderbilt University Medical Center in Nashville, TN; Northwestern Memorial Hospital in Chicago, IL; Christiana Hospital and Wilmington Hospital in Wilmington, DE. Nearly all are large academic tertiary care facilities. All participate in the International Cohort of Avoidable REadmissions (ICARE) consortium. ${ }^{9}$ The non-US hospitals that are a part of ICARE were excluded from this analysis given the focus on conditions targeted by the HRRP specific to the United States. We collected data from the electronic health record of individual hospitals because calculation of the HOSPITAL score requires knowledge of laboratory studies, prior admissions, and primary treating service; these factors are not found in national samples of hospital discharges. As the HOSPITAL score was originally derived and validated in medical inpatients (surgical patients were excluded) this study was similarly restricted to patients discharged from an inpatient medical service.

Patients were excluded if they transferred to another inpatient health care facility (hospital or psychiatric hospital), discharged against medical advice, or had a hospital length of stay of $<1$ day. The study had Institutional Review Board approval from each site and the managing site at Brigham and Women's Hospital in Boston, Massachusetts.

\section{Identifying Potentially Preventable Readmissions}

All 30-day readmissions to any division of these hospitals were identified first. As the unit of analysis was a hospital discharge, a discharge could be considered an index admission and a readmission if several hospital stays occurred within 30 days. Potentially preventable readmissions from this cohort were identified using the SQLape algorithm as was performed in studies validating the HOSPITAL score. ${ }^{8,9}$ This algorithm uses administrative data to categorize readmissions as "unavoidable" or "potentially preventable." $8,11,12$ The SQLape algorithm was developed from physician adjudication of $>3000$ reviewed patient charts, then validated in $>130,000$ patients across 49 hospitals, including a 12-hospital chart review study for validation. ${ }^{11}$ It uses a similar method to CMS for determining "planned" readmissions, but goes further to identify other unavoidable and avoidable hospitalizations. ${ }^{13,14}$ Thus, "potentially preventable" readmissions determined by SQLape would be considered unplanned readmissions by CMS, but not all unplanned readmissions would be considered "potentially preventable" by SQLape. Further details about the SQLape algorithm are listed in the Supplemental Appendix (Supplemental Digital Content 1, http://links.lww.com/MLR/ B303).

As a sensitivity analysis, we also evaluated performance of the HOSPITAL score on all readmissions (not just those considered potentially preventable); in this analysis any patient with a 30-day readmission was compared with those without a readmission in this time frame. This is similar to the CMS measure. We also conducted a sensitivity analysis restricting to patients 65 years of age or above as a proxy for Medicare beneficiary status as we were unable to capture payor source as a part of our dataset and Medicare claims lacked the requisite data to calculate the HOSPITAL score.

\section{Calculating the HOSPITAL Score}

The development of the HOSPITAL score has been previously described. ${ }^{8}$ Briefly, it was originally derived and internally validated at a single academic US hospital in a sample of discharged medical inpatients. ${ }^{8}$ Logistic regression with backward selection was performed to identify the most significant predictors of potentially preventable readmissions within 30 days of discharge (Table 1). Score points were attributed to each of the 7 predictive variables identified using a regression coefficient-based scoring method. It was then validated in an international sample of all medical inpatients discharged from 9 hospitals from 4 countries (the ICARE consortium, which was created initially for this purpose), involving $>110,000$ records. ${ }^{9}$ This study represents a subsample of this validation study, restricted to the US hospitals that participated.

The HOSPITAL score (Table 1) includes 7 variably weighted predictor variables associated with potentially preventable 30-day readmissions in derivation and validation studies. ${ }^{8,9}$ These variables include assessment of the number of hospital admissions in the prior 12 months, urgency of admission (urgent or emergent vs. nonemergent), last avail- 
TABLE 1. HOSPITAL Score Calculation

\begin{tabular}{lcc}
\hline Characteristics & Value & Points \\
\hline Low Hemoglobin level at discharge $(<12 \mathrm{~g} / \mathrm{dL})$ & Yes & 1 \\
Discharge from an Oncology service & Yes & 2 \\
Low Sodium level at discharge $(<135 \mathrm{mmol} / \mathrm{L})$ & Yes & 1 \\
Procedure during hospital stay & Yes & 1 \\
Index admission Type: urgent or emergent & Yes & 1 \\
No. hospital Admissions in prior year & $\leq 1$ & 0 \\
& $2-5$ & 2 \\
& $\geq 5$ & 5 \\
Index hospitalization Length of stay $\geq 5 \mathrm{~d}$ & Yes & 2 \\
\hline
\end{tabular}

The HOSPITAL score has 13 total points as scored above.

Bold indicates the letters associated with the HOSPITAL acronym from each characteristic.

able laboratory studies (hemoglobin and sodium levels), discharge from an oncology division, any medical procedures or complex imaging performed during the index hospitalization, and the index hospital length of stay. As only nonsurgical patients were enrolled, medical procedures included those traditionally performed by a medical physician, such as endoscopy, hemodialysis, transfusion, percutaneous coronary interventions, or bedside procedures like paracentesis or thoracentesis. Complex imaging studies included computed tomographic scans or magnetic resonance imaging. Laboratory studies were the only missing data in this analysis; of 78,921 patients in the cohort, 6259 had missing hemoglobin values, $7.9 \%$, and 3076 had missing sodium values, $3.9 \%$. This means that these laboratory studies were not measured during the course of the hospitalization in question; thus, we have assumed that the patient did not have these risk factors.

The scoring system ranges from 0 to 13 points with higher scores connoting higher risk of readmission. These risks were further categorized into 3 groups: low risk (up to 4 points); intermediate risk (5-6 points); and high risk ( 7 or more points), roughly corresponding to $5 \%, 10 \%$, and $20 \%$ risk of potentially preventable 30 -day readmissions, respectively. 8,9

\section{Measuring HOSPITAL Score Performance}

We used a multivariable logistic regression model including the HOSPITAL score with a fixed effect for hospitals to account for differences in outcomes across hospitals. We used a robust sandwich variance estimator to account for repeated events by the same patient within hospitals. We calculated the overall performance, the discriminatory power, and the calibration of this model for each of the medical diagnoses targeted by the HRRP: acute myocardial infarction, chronic obstructive pulmonary disease (COPD), pneumonia, or heart failure (HF). We used Medicare's methodology for identifying these conditions as described in the 2015 Final Rule. ${ }^{14}$

We used the Brier score to quantify accuracy, or how close predictions are to the actual outcome. Scores range from 0 (best performance) to 0.25 (noninformative). We calculated the $c$-statistic to evaluate discrimination; that is, the ability of the HOSPITAL score to discriminate between those with a potentially preventable 30 -day readmission and those without a potentially preventable 30-day readmission. The $c$-statistic was obtained through a logistic regression model that included all variables in the HOSPITAL score, whereas the calibration evaluates how closely the predicted readmission rate matches the true observed rate within each of the 3 risk score categories described above. Finally, we used the Hosmer-Lemeshow goodness-of-fit test to measure calibration of the model, reporting observed and predicted rates for each deciles of risk; a $P \geq 0.05$ implies a good fit, with higher numbers signifying better fit. ${ }^{15}$ Analyses were performed with SAS Software, version 9.3 (SAS Institute Inc., Cary, NC).

\section{RESULTS}

Of 78,921 discharges during the study period, 9181 $(11.6 \%)$ were discharged after treatment for one of the HRRP-targeted conditions [pneumonia $(n=3335,4.2 \%)$, HF $(\mathrm{n}=3189,4.0 \%)$, COPD $(\mathrm{n}=1890,2.4 \%)$, acute myocardial infarction $(\mathrm{n}=767,1.0 \%)]$. The potentially avoidable readmission rate across these diagnoses was $13.6 \%$ overall (Fig. 1). Patients treated for HRRP-targeted conditions who had potentially preventable readmissions were more likely to have low sodium and hemoglobin levels and to have more prior admissions in the past year (Table 2).

Across all 4 diagnoses, the HOSPITAL score had very good accuracy (Brier score $=0.11$ ) good discrimination $[c$ statistic $=0.68(95 \% \mathrm{CI}, 0.66-0.70)]$, and very good calibration (Hosmer-Lemeshow goodness-of-fit $P=0.77$ ). For example, the expected and observed readmission rates were very similar within each risk subgroup: low risk $(9.1 \%$ expected, 9.6\% observed), moderate risk (11.3\% expected, $11.0 \%$ observed), and high risk (18.0\% expected, $18.1 \%$ observed). Within diagnoses, accuracy and discrimination were similar (Brier score, 0.10-0.12; $c$-statistic, 0.67-0.71), although calibration was better for pneumonia and COPD $(P=0.76$ and 0.81 , respectively) than for acute $\mathrm{MI}$ or $\mathrm{HF}$ $(P=0.16$ and 0.17 , respectively-Table 3$)$. Results were similar when evaluating only patients age 65 and above (Table 4) and when considering all readmissions (not just those considered potentially preventable-Table 5).

\section{DISCUSSION}

In this multicenter retrospective cohort, the HOSPITAL score had very good accuracy, discrimination, and calibration for 30-day potentially preventable readmissions after inpatient treatment for 1 of the 4 medical conditions targeted by the Hospital Readmission Reduction Program (HRRP). These findings are important for practitioners and hospitals seeking to reduce preventable readmissions and improve patient outcomes.

The HOSPITAL score may fill a needed niche as a risk prediction score that is validated in multiple institutions in the United States and abroad, and identifies a high-risk population for potentially avoidable admissions. The HOSPITAL score has better performance characteristics than most prediction models using retrospective data in all-cause readmissions and within specific disease states, separating the HOSPITAL score from prior risk prediction models. ${ }^{16}$ In 


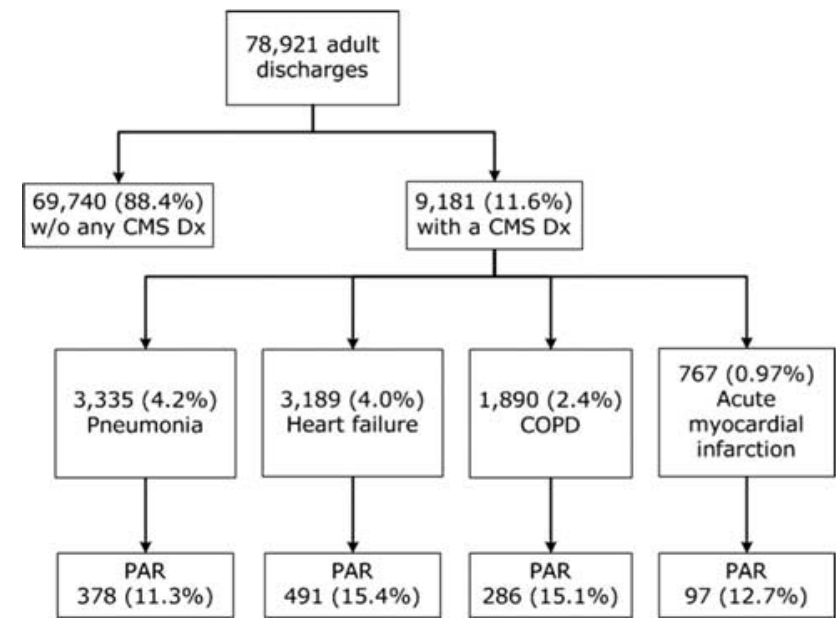

FIGURE 1. Cohort description and derivation. CMS $\mathrm{dx}$ indicates 1 of the 4 medical conditions targeted by the Medicare Hospital Readmission Reductions Program; COPD, chronic obstructive pulmonary disease; PAR, potentially avoidable readmission. The number of patients (and proportion of the overall total) are listed.

particular, our results suggest that the HOSPITAL score can be used both to support comprehensive transitional care interventions which have a track record of success, ${ }^{17,18}$ or disease-specific interventions within diagnoses penalized as a part of the HRRP. Examples of integrating a risk prediction score into an intervention are preliminary, ${ }^{19}$ but suggest such a model tailors the intervention to those most at risk, offering the most promise of reducing readmissions in a maximally cost-effective manner.
Although the HOSPITAL score can help predict a high-risk cohort for readmission and segregates out likely nonpreventable readmissions, it does not explain why the patient might be readmitted or how to intervene to reduce readmission risk. Many of the markers in the score (such as previous hospital admissions) are clearly not modifiable, and others (low hemoglobin) are likely associated with sicker patients rather than representing targets for intervention. Rather, the score could be used to identify a high-risk cohort, who then could be formally assessed for risk factors for readmission that might be modifiable, and receive a tailored intervention. ${ }^{4,6}$ Surveys of hospital administrators indicate that they use both disease-specific and more general interventions to reduce readmissions. ${ }^{20-22}$ A relative weakness of the HOSPITAL score in this regard is that it requires data available shortly before discharge (length of stay and laboratory studies) which may ultimately affect which risk group the patient fits into. However, clinicians may find that the length of stay and likely ranges for these laboratory studies can often be predicted within 1-2 days before discharge.

The proportion of HRRP-targeted conditions in our sample was similar to other national data. ${ }^{23}$ Despite targeting a minority of conditions, readmission rates may be improving nationally both for targeted and nontargeted conditions ${ }^{23-25}$; Medicare is adding additional conditions each year. $^{26}$

Although a full discussion of the relative preventability of readmissions is beyond the scope of this paper, ${ }^{27-29}$ we note recent studies assessing preventability in the eye of patients and providers document high rates of potentially preventable readmissions, although these still constitute a minority of readmissions overall. ${ }^{30-32}$ In our cohort, the

TABLE 2. Baseline Characteristics of Patients Treated for HRRP-targeted Condition Stratified by 30-Day Potentially Preventable Readmission

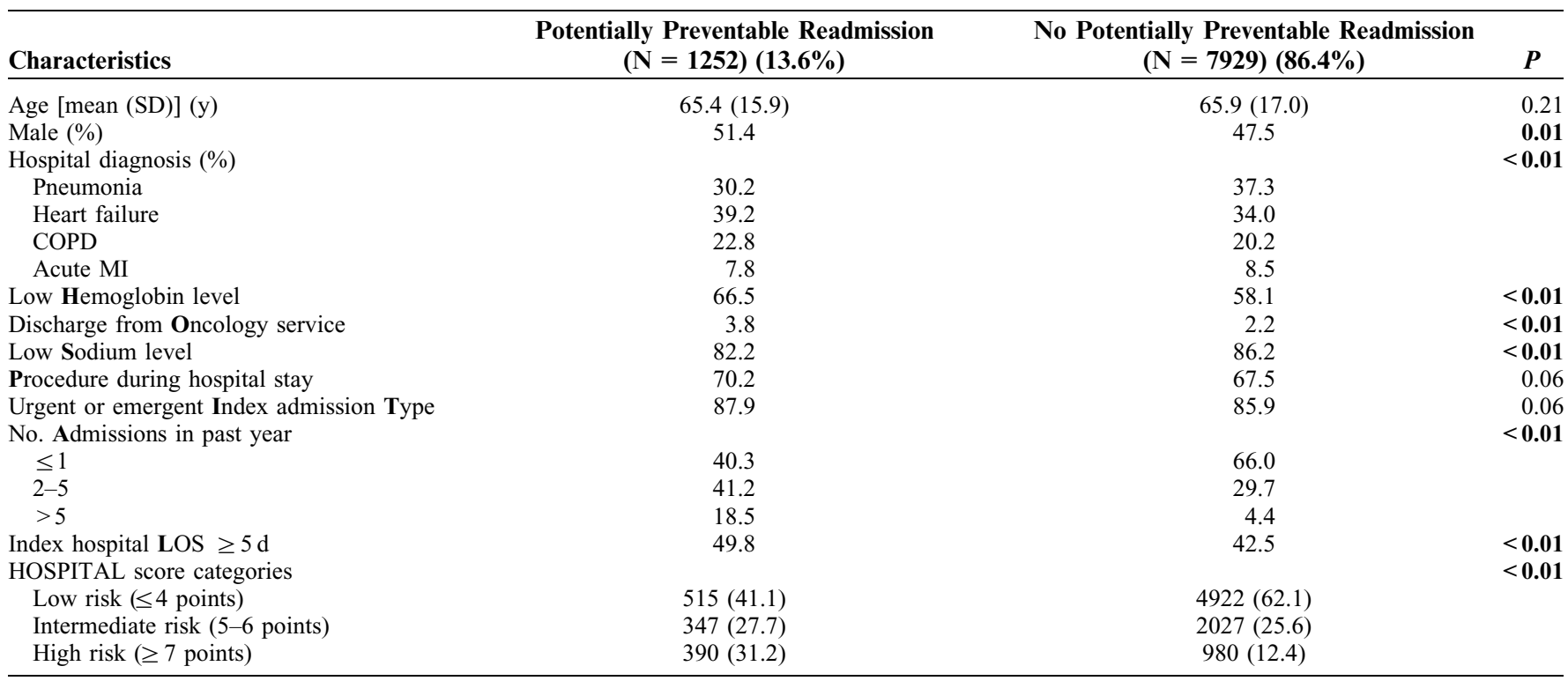

Bold signifies letters comprising the HOSPITAL score and statistically significant $P$-values.

COPD indicates chronic obstructive pulmonary disease; HRRP, Hospital Readmissions Reduction Program; LOS, length of stay; MI, myocardial infarction. 
TABLE 3. HOSPITAL Score Performance in Potentially Preventable 30-Day Readmissions

\begin{tabular}{lccccc}
\hline Characteristics & Discharges (N) & 30-Day Readmission Rate (\%) & Brier Score & $\boldsymbol{c}$-Statistic (95\% CI) & Hosmer-Lemeshow (P) \\
\hline All CMS diagnoses & 9181 & 13.6 & 0.11 & $0.68(0.66-0.70)$ & 0.77 \\
Pneumonia & 3335 & 11.3 & 0.10 & $0.68(0.65-0.71)$ & 0.76 \\
Heart failure & 3189 & 15.4 & 0.12 & $0.68(0.65-0.70)$ \\
Acute MI & 767 & 12.7 & 0.11 & $0.67(0.61-0.72)$ \\
COPD & 1890 & 15.1 & 0.12 & $0.71(0.67-0.74)$ & 0.17 \\
\hline
\end{tabular}

CI indicates confidence interval; CMS, Center of Medicare and Medicaid Services; COPD, chronic obstructive pulmonary disease; MI, myocardial infarction.

preventable readmission rate was lower than in these studies. This may reflect the difference between using administrative data versus interviews or comprehensive chart review of readmissions to determine preventability. It should be noted that there is currently no consensus on how to identify with certainty which readmissions are truly preventable, no matter how laborious the process. Not all potentially preventable readmissions identified through administrative data and/or electronic health records (such as those identified by SQLape) may be preventable. For example, in SQLape's largest validation study, the true positive rate for identifying potentially preventable readmissions was $78 \%$ based on medical chart review, with most errors related to coding rather than errors in the algorithm. ${ }^{11}$ However, we feel the advantages of SQLape-including its transparent design, large multisite derivation, and validation samples with chart review substudies for validation - outweigh potential misclassification bias, particularly compared with the CMS allcause readmission measure (which does not attempt to identify any readmissions as potentially preventable) or other tools to assess preventable readmissions using administrative data, which have not been externally validated. ${ }^{33}$ Many hospitals and providers will seek to identify an enriched cohort of potentially avoidable readmissions using administrative data, a niche the HOSPITAL score may help to fill.

This study should be considered in the context of the data analyzed. As the HOSPITAL score was derived and validated in medical patients, it has not been studied in surgical populations or for surgical diagnoses (eg, joint replacement) targeted by the HRRP. Although very few patients were coded as being discharged from an oncology service, none of the HRRP-targeted measures is primarily associated with an oncologic diagnosis, raising concern about whether this coding was accurate. Anecdotally, oncology services at some hospitals in our sample also admit their own patients for acute medical concerns, not just for scheduled chemotherapy, potentially explaining this finding.

The HOSPITAL score does not include several important predictors of hospital readmission, such as functional status, ${ }^{32,33}$ socioeconomic status, ${ }^{34}$ or hospital characteristics. $^{24}$ However, these characteristics are infrequently captured in administrative data, and the HOSPITAL score was purposefully limited to 7 variables to increase clinical applicability and ease of use. Diagnoses treated are subject to coding error, and we were unable to conduct chart review to confirm coding accuracy or validate readmissions flagged as potentially avoidable using the SQLape algorithm. Although the original score was validated in 9 centers across 4 countries, this analysis was limited to predominantly large academic medical centers, and the predictive characteristics of the HOSPITAL score may be different in other settings for HRRP-targeted conditions.

We did not compare the performance of the HOSPITAL score with CMS's risk-adjustment model for hospital readmissions as the intent of the 2 models is different. The HOSPITAL score was derived and validated to predict 30 day potentially avoidable readmissions, whereas CMS's riskadjustment measure was developed to enable comparison of readmission rates across hospitals through adjustment for patient readmission risk factors that may be unequally distributed across hospitals. We also did not attempt to derive a new risk prediction score for HRRP-targeted conditions, but rather to analyze the performance of the original HOSPITAL score in these specific populations. Thus, while some predictors that were valid in the original derivation study did not meet statistical significance and other predictors could have better performance in these select populations, the overall value of the HOSPITAL score lies in its prediction of potentially preventable readmissions across medical inpatients in a variety of contexts, as well as its performance

TABLE 4. HOSPITAL Score Performance in Potentially Preventable 30-Day Readmissions Restricted to Patients Age 65 Years or Above

\begin{tabular}{lccccc}
\hline Characteristics & Discharges (N) & 30-Day Readmission Rate (\%) & Brier Score & $\boldsymbol{c}$-Statistic (95\% CI) & Hosmer-Lemeshow (P) \\
\hline All CMS diagnoses & 5087 & 13.2 & 0.11 & $0.66(0.63-0.68)$ \\
Pneumonia & 1717 & 11.8 & 0.10 & $0.64(0.60-0.69)$ & 0.27 \\
Heart failure & 1918 & 14.1 & 0.12 & $0.68(0.64-0.71)$ & 0.27 \\
Acute MI & 421 & 12.4 & 0.12 & $0.68(0.61-0.76)$ & 0.37 \\
COPD & 1031 & 14.1 & 0.10 & $0.67(0.62-0.72)$ & 0.56 \\
\hline
\end{tabular}

CI indicates confidence interval; CMS, Center of Medicare and Medicaid Services; COPD, chronic obstructive pulmonary disease; MI, myocardial infarction. 
TABLE 5. HOSPITAL Score Performance in All 30-Day Readmissions

\begin{tabular}{lccccc}
\hline Characteristics & Discharges $(\mathbf{N})$ & 30-Day Readmission Rate (\%) & Brier Score & $\boldsymbol{c}$-Statistic (95\% CI) & Hosmer-Lemeshow $(\boldsymbol{P})$ \\
\hline All CMS diagnoses & 9181 & 16.3 & 0.13 & $0.68(0.66-0.69)$ & 0.41 \\
Pneumonia & 3335 & 13.8 & 0.13 & $0.68(0.65-0.70)$ & 0.49 \\
Heart failure & 3189 & 18.2 & 0.13 & $0.67(0.65-0.70)$ & 0.10 \\
Acute MI & 767 & 17.4 & 0.13 & $0.66(0.61-0.71)$ \\
COPD & 1890 & 16.6 & 0.13 & $0.70(0.67-0.73)$ & 0.49 \\
\hline
\end{tabular}

CI indicates confidence interval; CMS, Center of Medicare and Medicaid Services; COPD, chronic obstructive pulmonary disease; MI, myocardial infarction.

for HRRP-targeted conditions. A single score that performs well in all contexts has more utility than multiple individualized scores specialized to specific contexts.

The HOSPITAL score is a valid risk prediction model for 30-day potentially preventable readmissions across all readmissions and within important disease-specific categories. As such, it may serve as a key part of future costeffective interventions to reduce preventable readmissions.

\section{REFERENCES}

1. Berenson RA, Paulus RA, Kalman NS. Medicare's readmissionsreduction program - a positive alternative. $N$ Engl J Med. 2012;366: 1364-1366.

2. Brown JR, Sox HC, Goodman DC. Financial incentives to improve quality: skating to the puck or avoiding the penalty box? JAMA. 2014;311:1009-1010.

3. Boccuti C, Casillas G. Aiming for fewer hospital U-turns: he Medicare Hospital Readmission Reduction Program. 2015. Available at: http:// kff.org/medicare/issue-brief/aiming-for-fewer-hospital-u-turns-the-medicare-hospital-readmission-reduction-program/. Accessed April 25, 2016.

4. Burke RE, Coleman EA. Interventions to decrease hospital readmissions: keys for cost-effectiveness. JAMA Intern Med. 2013;173: 695-698.

5. Leppin AL, Gionfriddo MR, Kessler M, et al. Preventing 30-day hospital readmissions: a systematic review and meta-analysis of randomized trials. JAMA Intern Med. 2014;174:1095-1107.

6. Burke RE, Kripalani S, Vasilevskis EE, et al. Moving beyond readmission penalties: creating an ideal process to improve transitional care. J Hosp Med Off Publ Soc Hosp Med. 2013;8:102-109.

7. Burke RE, Guo R, Prochazka AV, et al. Identifying keys to success in reducing readmissions using the ideal transitions in care framework. BMC Health Serv Res. 2014;14:423. doi:10.1186/1472-6963-14-423.

8. Donzé J, Aujesky D, Williams D, et al. Potentially avoidable 30-day hospital readmissions in medical patients: derivation and validation of a prediction model. JAMA Intern Med. 2013;173:632-638.

9. Donzé JD, Williams MV, Robinson EJ, et al. International validity of the HOSPITAL score to predict 30-day potentially avoidable hospital readmissions. JAMA Intern Med. 2016;176:496-502.

10. Hansen LO, Young RS, Hinami K, et al. Interventions to reduce 30-day rehospitalization: a systematic review. Ann Intern Med. 2011;155: 520-528.

11. Halfon P, Eggli Y, Prêtre-Rohrbach I, et al. Validation of the potentially avoidable hospital readmission rate as a routine indicator of the quality of hospital care. Med Care. 2006;44:972-981.

12. Halfon P, Eggli Y, van Melle G, et al. Measuring potentially avoidable hospital readmissions. J Clin Epidemiol. 2002;55:573-587.

13. Eggli Y. SQLape algorithm. 2014. Available at: www.sqlape.com/ AR_ALGORITHM.htm. Accessed August 26, 2016.

14. Centers for Medicare and Medicaid Services. Measure methodology. 2016. Available at: www.cms.gov/Medicare/Quality-Initiatives-PatientAssessment-Instruments/HospitalQualityInits/Measure-Methodology.html. Accessed August 26, 2016.

15. Steyerberg EW, Vickers AJ, Cook NR, et al. Assessing the performance of prediction models: a framework for some traditional and novel measures. Epidemiology. 2010;21:128-138.
16. Kansagara D, Englander H, Salanitro A, et al. Risk prediction models for hospital readmission: a systematic review. JAMA. 2011;306: 1688-1698.

17. Coleman EA, Parry C, Chalmers S, et al. The care transitions intervention: results of a randomized controlled trial. Arch Intern Med. 2006;166:1822-1828.

18. Naylor MD, Brooten D, Campbell R, et al. Comprehensive discharge planning and home follow-up of hospitalized elders: a randomized clinical trial. JAMA. 1999;281:613-620.

19. Amarasingham R, Patel PC, Toto K, et al. Allocating scarce resources in real-time to reduce heart failure readmissions: a prospective, controlled study. BMJ Qual Saf. 2013;22:998-1005.

20. Vasilevskis EE, Kripalani S, Ong MK, et al. Variability in implementation of interventions aimed at reducing readmissions among patients with heart failure: a survey of teaching hospitals. Acad Med. 2016; 91:522-529.

21. Bradley EH, Curry L, Horwitz LI, et al. Contemporary evidence about hospital strategies for reducing 30-day readmissions: a national study. J Am Coll Cardiol. 2012;60:607-614.

22. Brewster AL, Cherlin EJ, Ndumele CD, et al. What works in readmissions reduction: how hospitals improve performance. Med Care. 2016;54:600-607.

23. Zuckerman RB, Sheingold SH, Orav EJ, et al. Readmissions, observation, and the hospital readmissions reduction program. $N$ Engl J Med. 2016;374:1543-1551.

24. Joynt KE, Orav EJ, Jha AK. Thirty-day readmission rates for Medicare beneficiaries by race and site of care. JAMA. 2011;305:675-681.

25. McGarry BE, Blankley AA, Li Y. The impact of the Medicare hospital readmission reduction program in New York state. Med Care. 2016;54:162-171.

26. Centers for Medicare and Medicaid Services. Readmissions-ReductionProgram. 2016. Available at: www.cms.gov/medicare/medicare-fee-forservice-payment/acuteinpatientpps/readmissions-reduction-program.html. Accessed April 27, 2016.

27. Lavenberg JG, Leas B, Umscheid CA, et al. Assessing preventability in the quest to reduce hospital readmissions. J Hosp Med. 2014;9: 598-603.

28. van Walraven C, Jennings A, Taljaard M, et al. Incidence of potentially avoidable urgent readmissions and their relation to all-cause urgent readmissions. CMAJ. 2011;183:E1067-E1072.

29. van Walraven C, Bennett C, Jennings A, et al. Proportion of hospital readmissions deemed avoidable: a systematic review. CMAJ. 2011;183: E391-E402.

30. Auerbach AD, Kripalani S, Vasilevskis EE, et al. Preventability and causes of readmissions in a national cohort of general medicine patients. JAMA Intern Med. 2016;176:484-493.

31. Ouslander JG, Naharci I, Engstrom G, et al. Root cause analyses of transfers of skilled nursing facility patients to acute hospitals: lessons learned for reducing unnecessary hospitalizations. J Am Med Dir Assoc. 2016; 17:256-262.

32. Ouslander JG, Naharci I, Engstrom G, et al. Lessons learned from root cause analyses of transfers of skilled nursing facility (SNF) patients to acute hospitals: transfers rated as preventable versus nonpreventable by SNF staff. J Am Med Dir Assoc. 2016;17:596-601.

33. Goldfield NI, McCullough EC, Hughes JS, et al. Identifying potentially preventable readmissions. Health Care Financ Rev. 2008;30:75-91.

34. Kind AJH, Jencks S, Brock J, et al. Neighborhood socioeconomic disadvantage and 30-day rehospitalization: a retrospective cohort study. Ann Intern Med. 2014;161:765-774. 